\title{
Solar Energy as an Energy Source: An Approach
}

\author{
Rucha V. Moharir ${ }^{1}$, Satyaprakash S. Dodani ${ }^{2}$ \\ ${ }^{1}$ Assistant Professor, P.I.G.C.E Nagpur, \\ ${ }^{2}$ Student, GNIT,
}

\begin{abstract}
The paper deals with utilization \& possibilities of solar energy (photo-voltaic systems) and with transformation of this energy to chemical energy as well as its utilization in the surface treatment of metals by electrochemical processes.This technology can be widely applied in machine industry in the future due to cheap electrical energy generation. Next advantage of this electrical energy generation is the decrease of negative environmental impact. Surface treatments affect lifetime, serviceability, usability, availability and maintenance of equipment. The financial return from a solar energy investment is affected by multiple factors such as the fluctuation of solar radiation over time and the price of electricity. Solar energy has many benefits compared to fossil fuels. It is clean and green, non-polluting and everlasting energy. For this reason it has attracted more attention than other alternative sources of energy in recent years. Many energy economists say that solar energy is going to play an increasingly important role in all our lives. To highlight the importance of such a source of energy becomes not only important but also inevitable. This paper analyzes the determining factors of solar energy usage and also analyzes the benefit of the different solar energy devises.
\end{abstract}

Keywords: Solar energy, utility, benefits, drawbacks, current scenario.

\section{Introduction}

Solar energy is the ultimate source of energy from millions of years and it is renewable energy. This energy consists of radiant light and heat energy from the sun. Out of all energy emitted by sun only a small fraction of energy is absorbed by the earth. Just this tiny fraction of the sun's energy that hits the earth is enough to meet all our power needs. Using present solar techniques some of the solar energy reaching the earth is utilized for generating electricity etc. Even then the energy demand met by using solar energy is very less. Solar energy is the main prerequisite of the life on the Earth. Solar radiation is a direct source for generating heat, cold and power. Indirectly, it is possible to use solar energy through hydro power, wind energy, energy of sea waves, heat energy of environs and energy of biomass $[1,2]$

\section{Scarcity Of Natural Resources}

Whether economic growth can be sustained in a finite natural world is one of the earliest and most enduring questions in economic literature. In essence, the issue is whether technological progress and capital accumulation can overcome diminishing marginal returns to finite natural resources. The debate begins with the birth of economics as a separate discipline and continues to this day (Fig 1). Its intellectual roots still play a prominent and significant role. It is the topic of the two previous volumes on Scarcity and Growth published by Resources for the Future. While the general nature of the debate is unchanged, the focus and topics of discussion have evolved.

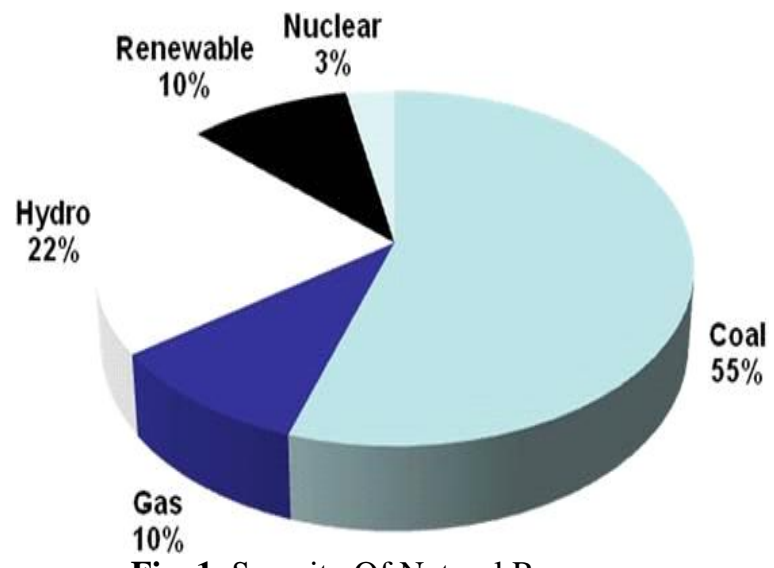

Fig. 1: Scarcity Of Natural Resources 
The earth's natural resources are finite, which means that if we use them continuously, we will eventually exhaust them. This basic observation is undeniable. But another way of looking at the issue is far more relevant to assessing people's well-being. Our exhaustible and non reproducible natural resources, if measured in terms of their prospective contribution to human welfare, can actually increase year after year, perhaps never coming anywhere near exhaustion. How can this be? The answer lies in the fact that the effective stocks of natural resources are continually expanded by the same technological developments that have fueled the extraordinary growth in living standards since the industrial revolution. The graphical representations of the wavelength entrapped by the different sources of sun are shown on the figure below Fig 2.

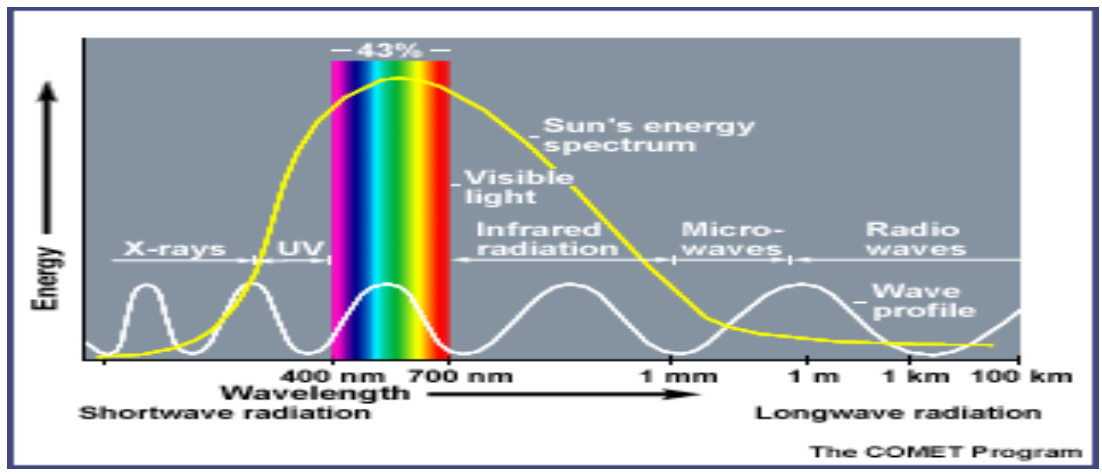

Fig 2: Graphical Representations Of The Wavelength

The surface receives about $47 \%$ of the total solar energy that reaches the Earth. Only this amount is usable.

\section{Benefits And Drawbacks Of Solar Energy}

All chemical and radioactive polluting by products of the thermonuclear reactions remain behind on the sun, while only pure radiant energy reaches the Earth. Energy reaching the earth is incredible. By one calculation, 30 days of sunshine striking the Earth have the energy equivalent of the total of all the planet's fossil fuels, both used and unused!

\subsection{Benefits}

1. Solar energy is free although there is a cost in the building of 'collectors' and other equipment required to convert solar energy into electricity or hot water.

2. Solar energy does not cause pollution. However, solar collectors and other associated equipment / machines are manufactured in factories that in turn cause some pollution.

3. Solar energy can be used in remote areas where it is too expensive to extend the electricity power grid.

4. Many everyday items such as calculators and other low power consuming devices can be powered by solar energy effectively.

5. It is estimated that the world's oil reserves will last for 30 to 40 years. On the other hand, solar energy is infinite (forever).

\subsection{Drawbacks}

1. Solar energy can only be harnessed when it is daytime and sunny.

2. Solar collectors, panels and cells are relatively expensive to manufacture although prices are falling rapidly.

3. Solar power stations can be built but they do not match the power output of similar sized conventional power stations. They are also very expensive.

4. In countries such as the UK, the unreliable climate means that solar energy is also unreliable as a source of energy. Cloudy skies reduce its effectiveness.

5. Large areas of land are required to capture the suns energy. Collectors are usually arranged together especially when electricity is to be produced and used in the same location.

6. Solar power is used to charge batteries so that solar powered devices can be used at night. However, the batteries are large and heavy and need storage space. They also need replacing from time to time. 


\section{Solar Energy Categorization}

There are two main categories of solar energy generation first one is solar thermal and the other one is solar photovoltaic.

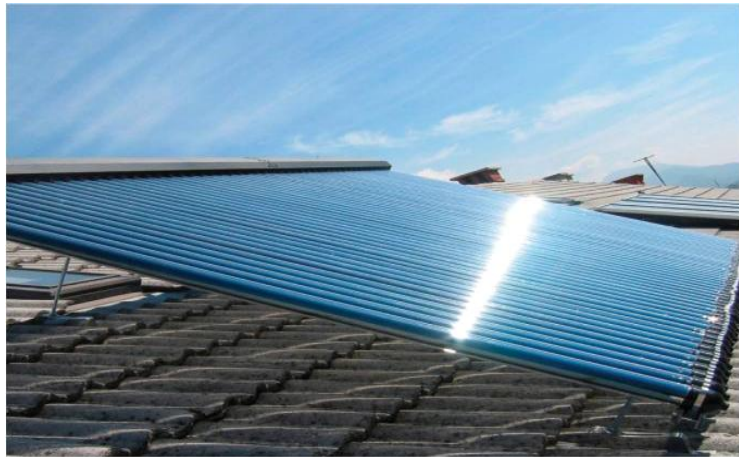

Fig 3: Solar Thermal

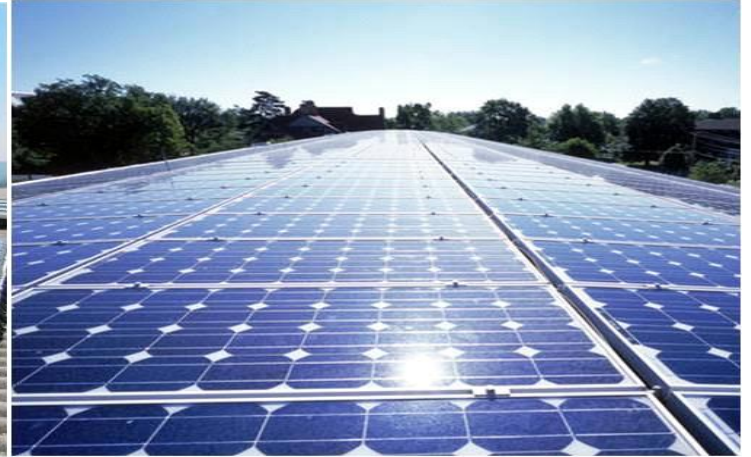

Fig 4: Solar Photovoltaic

$(\mathrm{PV})$

V. Solar Thermal Energy (STE)

Solar thermal energy (STE) is a form of energy and a technology for harnessing solar energy to generate thermal energy for use in industry, and in the residential and commercial sectors (Fig 3). The first installation of solar thermal energy equipment occurred in the Sahara Desert approximately in 1910 when a steam engine was run on steam produced by sunlight. Solar water heating is the most efficient and economical use of solar energy (Fig 5). Residential systems start at \$2500 and typically cost $\$ 3500-\$ 4500$ installed. Savings of \$30-\$75 per month, lasting 20 years. Tax credits and state rebates available.

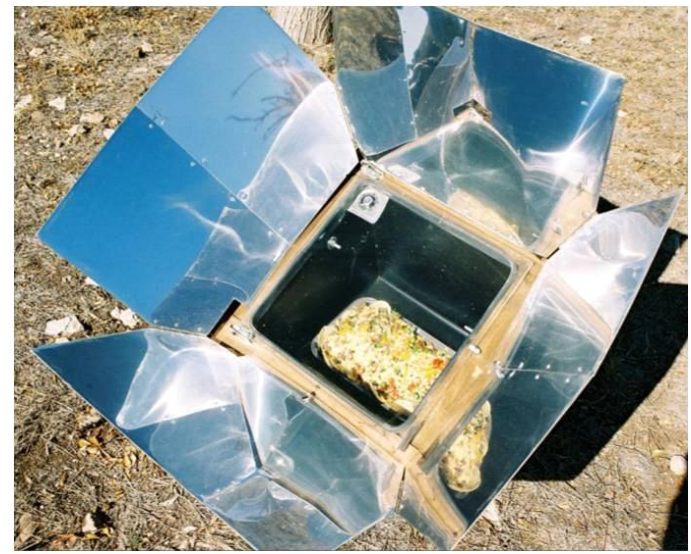

Fig 5: Solar Cooking

\section{Solar Water Heating System (SWH)}

SWH systems are generally very simple using only sunlight to heat water (Fig 6). A working fluid is brought into contact with a dark surface exposed to sunlight which causes the temperature of the fluid to rise. This fluid may be the water being heated directly, also called a direct system, or it may be a heat transfer fluid such as a glycol/water mixture that is passed through some form of heat exchanger called an indirect system. ${ }^{3}$ Solar water heating is the most efficient and economical use of solar energy. Residential systems start at $\$ 2500$ and typically cost $\$ 3500-\$ 4500$ installed. Savings of $\$ 30-\$ 75$ per month, lasting 20 years. Tax credits and state rebates available. 


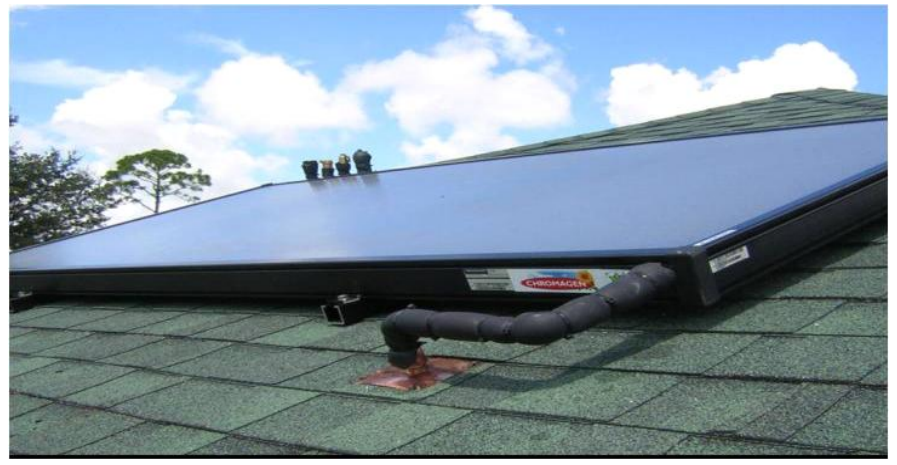

Fig 6: Water Heating

VII. Solar Photovoltaic (PV)

Solar energy is the main prerequisite of the life on the Earth. Solar radiation is a direct source for generating heat, cold and power. Indirectly, it is possible to use solar energy through hydropower, wind energy, energy of sea waves, heat energy of environs and energy of biomass [1].Thanks to photoelectric effect in semiconductors, we can transform the solar energy in solar cells to power energy. Transformation of solar energy to power energy has wide utilization (Fig 4). Disadvantage of solar energy generation is its dependence on daylight, season and cloudiness in the area. Even though, it is a potential energy, which should not be ignored [4].

\section{Solar Electric Systems}

Photovoltaic (PV) systems convert light energy directly into electricity. Commonly known as "solar cells." The simplest systems power the small calculators we use every day. More complicated systems will provide a large portion of the electricity in the near future. PV represents one of the most promising means of maintaining our energy intensive standard of living while not contributing to global warming and pollution. Solar cells are usually made of two thin pieces of silicon, the substance that makes up sand and the second most common substance on earth. One piece of silicon has a small amount of boron added to it, which gives it a tendency to attract electrons. It is called the p-layer because of its positive tendency. The other piece of silicon has a small amount of phosphorous added to it, giving it an excess of free electrons. There are various applications of solar photovoltaic like solar concentrators, centralized wind solar hybrid system, street lamp etc (Fig 7).

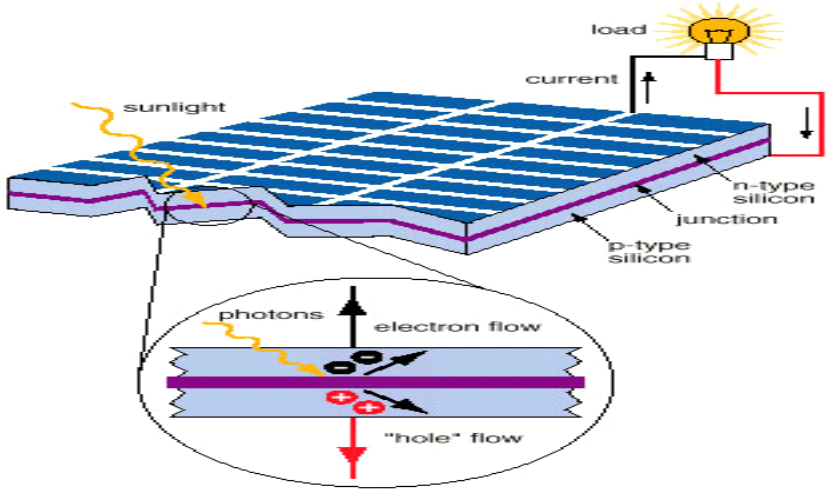

Fig 7: Photovoltaic (PV) systems

\subsection{Advantages}

\section{Advantages And Disadvantages Of Photovoltaic System (PV)}

Simple in application, relies on plastic bottles and sun light. Photo voltaic System reduces the risk of infectious diarrhea and dysentery from bacterial and parasitic infections and cholera. Its acceptability to users because of the simplicity of use. No cost to the users after obtaining the plastic bottles. Minimal change in taste of water. It is sustainable. The technique does not require consumables that are difficult or too expensive to obtain.

\subsection{Disadvantages}

Does not change chemical water quality. Requires relatively non turbid water for solar radiation to be effective in given time period of $6 \mathrm{~h}$. Need for pretreatment of water of higher turbidity not effective for very cloudy weather. Not as effective as boiling. SODIS processed water is not advisable for babies less than 18 months. The 
small treated volume (typically $\sim 2$ litres) associated with the PET bottles that are usually used in solar water disinfection (SODIS) is a major obstacle to its uptake in the developing world. SODIS is user dependent in that it requires the user to 'time' the exposure and as such there is no quality assurance in the process and as such, lack of compliance with the recommended protocol is a major issue.

\section{Case Studies}

\subsection{A Stand Alone Single Axis Offline PV Tracker Using Low Cost CMOS Circuitry[5]}

Ali Al-Mohamad calculated a Sun-tracking system, whereby the movement of a photovoltaic module was controlled to follow the Sun's radiation using a programmable logic-controller (PLC) unit. In this work, the designs for two axes and one axis tracking systems were performed using an open loop method of control based upon programmable logic controllers (PLC). Mohanad switching mode power-supply for the Sun-follower system Controls the tracking system movement. Monitors the PLC inputs and outputs: the main duty of this subroutine is to copy the status of the PLC inputs. In the present study, the azimuth and solar altitude angles of the sun were calculated for a period of 1 year at $37.6^{\circ}$ latitude in the Northern hemisphere, where Turkey is located, and according to these angles, an electromechanical system which tracks the sun on both of authors used a closed loop method of control to design sun tracking systems.

\subsection{Bright Copper Plating Using Photovoltaic as an Energy Source [6]}

The work deals with utilization opportunities of solar energy (photovoltaic systems) and with transformation of this energy to chemical energy as well as its application in the surface treatment of metals by electrochemical processes. Surface treatments expressively contribute to the resulting quality of technical equipment. This technology can be widely applied in machine industry in the future due to cheap electrical energy generation. Surface treatments affect lifetime, usability, serviceability, availability and maintenance of equipment. Next benefit of this electrical energy generation is the decrease of negative environmental impact. The whole system is now usable for bright copper plating, but owing to the low capacity, we can use it only for the objects of small areas, around $1.10 \mathrm{dm}^{2}, 4.39 \mathrm{dm}^{2}$ in ideal conditions.

\subsection{B. Leo Kwak, and JG Gordon II," 2-D mathematical modeling for a large electrochromic window Part I," Solar Energy Materials and Solar Cells [7]}

In this work, we describe a 2D time-dependent finite element based solver, established to simulate large area Li ion electrochromic devices. Electrochromic (EC) devices show a promise to be the next major advance in the energy-efficient window technology. However, the development of higher value (performance and cost) EC windows is the key to promote the applications of these energy saving devices. To that end, computer modeling may play a powerful role in providing in-depth understanding in EC device design, performance enhancement, material selection and development of EC layers. The results of 2D-model development and corresponding simulations are presented utilizing literature-based material properties. The capability of the model is established in treating a very large width-to-thickness aspect ratio and examining the impressions of a voltage change due to the spreading resistance and a diffusivity variation inside electrodes on device performance and lithium ion transport kinetics.

\subsection{Efficient conversion of solar energy to biomass and electricity [8]}

The Earth receives around 1000 W.m-2 of power from the Sun and only a fraction of this light energy is able to be converted to biomass (chemical energy) via the process of photosynthesis. Electrical energy can also be produced from this same solar resource via the use of photovoltaic modules. In this work we propose a novel method of combining both of these energy production processes to make full utilization of the solar spectrum and increase the productivity of light-limited microalgae systems. Out of all photosynthetic organisms, microalgae, due to their fast growth rates and their ability to grow on non-arable land using saline water, have been identified as potential source of raw material for chemical energy production. These two methods of energy production would appear to compete for use of the same energy resource (sunlight) to produce either chemical or electrical energy. Nevertheless, some groups of microalgae (i.e. Chlorophyta) only require the blue and red portions of the spectrum whereas photovoltaic devices can absorb strongly over the full range of visible light. This suggests that a combination of the two energy production systems would allow for a full utilization of the solar spectrum allowing both the production of chemical and electrical energy from the one facility making efficient use of available land and solar energy. In this work we propose to introduce a filter above the algae culture to modify the spectrum of light received by the algae and redirect parts of the spectrum to generate electricity. The electrical energy generated by this approach can then be directed to running ancillary systems or producing extra illumination for the growth of microalgae. This work it is modeled an approach whereby the productivity of light-limited microalgae systems can be improved by at least $4 \%$ through using an LED array to increase the total amount of illumination on the microalgae culture. 


\section{Summery}

The sun is a powerful source that can help our planet by giving us clean, reusable energy to power our world. The use of this energy is free, does not create pollution, and if used wisely can help us become less dependent on other more costly and damaging forms of power. In this frame work the beneficial techniques and advantages of solar energy is highlighted by showcasing different applications where solar energy is used as a renewable source. The benefits of solar energy over different applications are already stated in this work also with some interesting facts about where it requires more attention to work with. The present work is more helpful for analyzing the effects of solar energy and its broad aspects and where it can be more suitable for reducing the load on the environmental. Implementations of some good material in accordance with the available source is the best technique to be adopted for reducing the utility of electric demands and where the source called as renewable source used to reduce the load and cost of the treatment. Disadvantages are also emphasized in the current work for further reducing the chances of errors and more efficiency to be attained.

\section{References}

[1] BERANOVSKÝ, J., TRUXA, J., et al. Alternative energy for your house. Brno: ERA, 2004. ISBN 80-86517-59-4.

[2] LUQUE, A., HEGEDUS, S. Handbook of Photovoltaic Science and Engineering. John Wiley \& Sons Ltd, 2003. ISBN 0-47149196-9.

[3] Kishan Patel et al. Review of solar water heating systems. 2012. IJAET,Vol. III(IV), E-ISSN 0976-3945.

[4] MESSENGER, R. VENTRE, J. Photovoltaic Systems Engineering.New York:CRC Press LLC, 2005. ISBN 0-8493-1793-2.

[5] Al-Mohamad, A Stand Alone Single Axis Offline PV Tracker Using Low Cost CMOS Circuitry.

[6] Jozef Fiala, Anna Michalíková. Bright Copper Plating using Photovoltaic as an Energy Source, 2011.

[7] Y. Liu, L. Sun, G. Sikha, J. Isidorsson, S. Lim, A. Anders, B. Leo Kwak, and JG Gordon II, "2-D mathematical modeling for a large electrochromic window Part I," Solar Energy Materials and Solar Cells, vol. 120, Part A, pp. 1-8, 2014. Corresponding author: Dr. B. Leo Kwak.

[8] David Parlevliet and Navid Reza Moheimani, Efficient conversion of solar energy to biomass and electricity, Parlevliet and Moheimani Aquatic Biosystems 2014, 10:4. 